\title{
Behavioral Economics: A Primer and Applications to the UN Sustainable Development Goal of Good Health and Well-Being
}

\author{
Robert Siegel ${ }^{1,2, * \mathbb{D}}$, Katelyn Gordon ${ }^{1}$ and Linda Dynan ${ }^{1,3}$ \\ 1 The Heart Institute, Department of Pediatrics, and James M. Anderson Center for Health Systems Excellence, \\ Cincinnati Children's Hospital, Cincinnati, OH 45229, USA; katelyn.gordon@cchmc.org (K.G.); \\ dynanl@nku.edu (L.D.) \\ 2 Department of Pediatics, College of Medicine, University of Cincinnati, Cincinnati, OH 45229, USA \\ 3 Department of Economics, Haile College of Business, Northern Kentucky University, \\ Highland Heights, KY 41099, USA \\ * Correspondence: bob.siegel@cchmc.org
}

Citation: Siegel, R.; Gordon, K.; Dynan, L. Behavioral Economics: A Primer and Applications to the UN Sustainable Development Goal of Good Health and Well-Being. Reports 2021, 4, 16. https://doi.org/10.3390/ reports4020016

Academic Editor: Toshio Hattori

Received: 16 April 2021

Accepted: 30 May 2021

Published: 4 June 2021

Publisher's Note: MDPI stays neutral with regard to jurisdictional claims in published maps and institutional affiliations.

Copyright: (c) 2021 by the authors. Licensee MDPI, Basel, Switzerland. This article is an open access article distributed under the terms and conditions of the Creative Commons Attribution (CC BY) license (https:// creativecommons.org/licenses/by/ $4.0 /)$.

\begin{abstract}
Behavioral economics (BE) is a relatively new field within economics that incorporates insights from psychology that can be harnessed to improve economic decision making with the potential to enhance good health and well-being of individuals and societies, the third of the United Nations Sustainable Development Goals. While some of the psychological principles of economic decision making were described as far back as the 1700s by Adam Smith, BE emerged as a discipline in the 1970s with the groundbreaking work of psychologists Daniel Kahneman and Amos Tversky. We describe the basic concepts of BE, heuristics (decision-making shortcuts) and their associated biases, and the BE strategies framing, incentives, and economic nudging to overcome these biases. We survey the literature to identify how BE techniques have been employed to improve individual choice (focusing on childhood obesity), health policy, and patient and healthcare provider decision making. Additionally, we discuss how these BE-based efforts to improve health-related decision making can lead to sustaining good health and well-being and identify additional health-related areas that may benefit from including principles of BE in decision making.
\end{abstract}

Keywords: behavioral economics; UNSDG 3; economic decision making in health care

\section{Introduction}

Behavioral economics (BE) is a relatively new field within the discipline of economics. It harnesses insights from psychology to improve economic decision making in ways that have the potential to enhance good health and well-being of both individuals and societies, the third of the United Nations Sustainable Development Goals (UNSDG). While some of the psychological principles of economic decision-making were described by Adam Smith as early as the 1700s, BE emerged as a discipline in the 1970s because of the groundbreaking work of psychologists Daniel Kahneman and Amos Tversky.

Although Aldieri and colleagues [1] find that inequality, inclusive of health inequality, can undermine well-being, Usai and colleagues note that studies over the past thirty years have successively supported that well-being is vital for the development of economies and societies [2]. Thus, this essay contributes to practice by bringing the principles of BE to a wider audience in a position to employ its concepts to improve health and well-being specifically as they could be used to support UNSDGs. We describe the basic concepts of $\mathrm{BE}$, how and why decision makers use heuristics (decision making shortcuts), the biases entailed, and BE strategies to overcome these biases (framing, incentives, and economic nudging) for improved decision making. We further survey the literature to identify how BE techniques have been employed in individual choice (focusing on childhood obesity), governments' health policy, and patient and healthcare provider decision making. Additionally, we discuss how these BE-based efforts to improve health-related decision 
making can lead to sustaining good health and well-being and identify additional healthrelated areas that may benefit from including principles of $\mathrm{BE}$ in decision making.

\section{Materials and Methods Review}

We first present a basic primer on BE. We then assess the literature that describes how $\mathrm{BE}$ is relevant to (1) individual choices, where we draw significantly on earlier work to summarize the literature of the application of $\mathrm{BE}$ to healthy food choice among children; (2) government policy for public health; and (3) patient, provider and health care systems in medical care decisions. We then discuss the application of BE to sustainable development goals [3].

Given that the intent of this study was a general review of behavioral economics and its application to healthcare rather than systematic review, a general search approach of the literature was used. The three authors searched Google Scholar with the search terms "Behavioral Economics" + "Healthcare" from years 2017 to 2021 (28,800 results) and PubMed with the search term "Behavioral Economics" all years $(11,627)$. The results were then reviewed by the authors for pertinent articles. Additionally, any other pertinent articles that were discovered in analyzing the literature were included.

\section{Background and Literature Review}

\subsection{Behavioral Economics Primer}

In this section, we discuss how behavioral economics (BE) differs from "traditional" or neoclassical economics, core principles of $\mathrm{BE}$ and key strategies of $\mathrm{BE}$ relevant to promoting and maintaining good health and well-being.

\subsubsection{Neoclassical Economics vs. BE}

Neoclassical economics is the study of optimal choice under conditions of scarcity. It adopts a welfare economics framework that assumes that individuals can determine what is best for themselves. Their preferences, as revealed by the choices that they make under the constraints that they face, lead to the best choices as determined by the affected decision makers' preferences. These revealed preferences exhibit three characteristics: completeness, which means that an individual can judge between two alternatives and rank them; transitivity, which means that the individual's preferences are non-circular when there are more than two alternatives; and independence, which means that if the options are all altered identically, the original ranking is maintained.

However, welfare-theory-based neoclassical economics maintains a number of underlying assumptions regarding individual rationality in decision making that are hard to maintain in the face of human experience. These include: consistent preferences, subjective expected utility, emotionless deliberation, unlimited cognitive ability, unlimited willpower, unlimited attention, and frame and context independence [4]. For example, the concept of consistent preferences seems to break down in the face of changing context. People will choose differently based on the presentation, both the order and placement, of options. For example, the placement of food items in cafeterias has a significant impact on the food chosen [5], likewise for products in supermarkets [6]. When the placement is changed, then peoples' preferences seem to change. Attention, willpower and cognitive ability are all known to have distributions within a society, as demonstrated in the "marshmallow experiment" [7], that are rationed like scarce resources and certainly are not unlimited, making the neoclassical "rational person" model assumptions more obviously violable [8]. Indeed, people are sometimes willing to pay others to help them change their behaviors to align with what they know to be the better choices that they are unable to implement on their own (e.g., smoking, drinking, dieting, and exercise coaches as on the commitment website https: / / www.stickk.com/ (accessed on 1 June 2021), see for other strategies to overcome low levels of will power) [9].

$\mathrm{BE}$ acknowledges the limitations experienced by people navigating complex environments with competing demands as they attempt to make decisions that will optimize their 
well-being. In particular, BE acknowledges that decisions tend to be suboptimal and prone to error, even from the decision-makers' own perspectives, when the decision-makers lack experience or have limited information, and/or the consequences of their decisions may not be immediately felt. BE explicitly takes into account the psychological concept of two competing thought systems experienced by individual decision-makers known as System 1 (automatic) and System 2 (reflective) [10,11]. "System 1 operates automatically and quickly, with little or no effort and no sense of voluntary control while System 2 requires that individuals allocate attention to effortful mental activities, including complex computations [11]". System 1 requires less cognitive effort; in economic terms, it is less (cognitively) costly and therefore is more likely to be employed by the decision-maker unless he/she/they exert effort to slow down and activate his/her/their reflective abilities. System 1 offers the path of least resistance and is why on average, more people will choose default options. Thus, $\mathrm{BE}$ recognizes that the path requiring the least effort is the one most likely chosen.

Decision makers often reduce their own cognitive burden (cost) by using heuristics (i.e., rules of thumb) that provide intuitively appealing answers. Although such heuristics are convenient, Kahneman and Tversky have found predictable types of biases leading people to decide sub-optimally that are built into these easy decision rules that emerge when the two thought systems are interacting [12]. These biases are anchoring, availability and representativeness. Examples of these biases are discussed in Section 3.2.2 "Applications of BE in Medicine" below.

A second set of errors that increase decision-makers vulnerability to biases occur when individuals operate in "hot states." These are periods when cognitive load is already high, the person is tired, hungry and/or the individual is in a distracting environment. All of these conditions reduce willpower, attention, and emotionless deliberation, thereby increasing reliance on System 1 thinking (reflexive) over System 2 thinking (deliberate). Decision-makers operating in hot states are especially prone to accept heuristics and their associated biases.

$\mathrm{BE}$ leverages these predictable patterns in human decision making that yield poor choices to overcome barriers to behavior change in ways that will improve the chances that the expected preferred choice will be the individual's choice. BE intentionally builds choice frameworks, presentations and deliberate messaging that reduce cognitive load so that the desired choice, from the perspective of the decision-maker, flows from the least costly thought process and is the one that is more likely to be selected [13]. This is how BE differs from the neoclassical economic tradition that assumes that individuals respond rationally to incentives alone and will accordingly make the best choice subject to the constraints they face without acknowledging that the choice framework and structures themselves come with biases and influences.

\subsubsection{Main Principles of BE Implementation}

"Freedom is not a contested value; all the participants in the debate are in favor of it" [11].

Thaler and Sunstein, academic proponents of using BE to influence decision-makers, introduced the concept "libertarian paternalism." Libertarian paternalism seeks to maintain individual freedom of choice while guiding individual choices to the ones that serve the decision makers' long-term interests through the use of nudges over mandates [10].

"A nudge is any aspect of the choice architecture that alters people's behavior in a predictable way without forbidding any options or significantly changing their economic incentives" [10].

These guiding principles mean that those in a position to present choices to individuals and society use what is known about predictable human behavior to make the "better" choice the easier choice. Thus, the architects of choice reduce the cognitive load of the better choice relative to suboptimal choices so that the best choice is associated with the least costly decision path. It employs framing and context to prime automatic reflexes to 
more closely align with the optimal choice. This includes, for example, making the better choice the default choice, requiring thought and action to opt out; signaling better choices heuristically (via visual clues) to reduce cognitive load; and reframing information to avoid pitfalls in intuitive reasoning and choice patterns.

\subsubsection{Key Strategies from BE}

Thaler and Sunstein contend that humans respond to incentives but they are influenced by nudges [10]. They provide a useful pneumonic for strategies associated with BE as implemented using libertarian paternalism through the use of nudges:

iNcentives

Understand mapping

Defaults

Give feedback

Expect error

Structure complex choices

Incentives, including prices, taxes and subsidies, are salient heuristics for decision making. BE can strengthen these traditional economic tools by incorporating prospect theory. This theory purports (and some evidence supports [14,15]; contrary that individuals are much more sensitive to losses than they are to gains of the same size [16]. Therefore, beyond the simple use of taxes and subsidies to favor particular behaviors such as soda taxes, cigarette taxes and employer-sponsored gym membership, these incentives could be structured as losses as opposed to gains. For example, suppose everyone receives a gym membership benefit. If the benefit goes unused in the first six months, it is lost. It is likely that many more people will head to the gym in the last week before eligibility is lost.

Understanding mapping requires that we consider the relationship between choice and the decision-makers' ultimate experience with that choice- that is, how does the choice translate (that is, map) to experience? Providing clearer information that compares options on price, quality and what is known about individual experience can enhance the decision-maker's ability to accurately assess the choice set and anticipate his/her/their expected utility more accurately.

Defaults are the outcomes that require no choice on the part of the decision-maker. With limited attention to discern differences in complex alternatives, setting appropriate defaults improves the quality of decisions in the individual's long-term interest by making the best choice the easiest, no-action one. Well-known examples of changing the default include automatic enrollment in health plans or employer-sponsored retirement savings plans while opting out of such plans requires deliberate effort.

"Give Feedback" suggests that visual evidence or confirmation that the decision maker is on the right path to an appropriate response/outcome can be provided. It can be accomplished by emoticons or other signaling labels.

"Expect error" refers to anticipating errors, as may be possible from the predictable errors associated with different heuristics and then designing choices to sidestep these potential errors.

Structure Complex Choices allows for making choices under uncertainty in two stages: editing and evaluating. Editing reduces the choice set by simplifying information for comparison, detecting and eliminating dominated choices, ignoring identical components across options (focusing on their differences) and assessing gains or losses. Evaluating then identifies and weights the salient characteristics as a gain or loss relative to a reference point rather than the absolute level of the outcomes that generate utility, thus taking into account prospect theory, where a gain generates less positive utility than the same loss would generate negative utility [17].

These sets of interventions offer a set of key strategies for improving decision making. However, BE strategies are not without their detractors. For example, Pesendorfer writes: "Behavioral economics emphasizes the context-dependence of decision making. A corollary of this observation is that it is difficult to extrapolate from experimental settings to field data 
or, more generally, economic settings. Moreover, not all variables that are shown to matter in some experiment are useful or relevant in economic applications. The question whether a particular variable is useful or even observable for economics rarely comes up in behavioral models, yet the success or failure of modeling innovations often depends on its answer" [18]. As another example, Kelman (1998) argues that behavioral economists would be wise to recognize the "dramatic limitations on the completeness of their theoretical accounts" [19].

Nonetheless, the next section details application of BE to support healthy choices by individual decision makers, to government involvement in public health and to medical care settings. It further provides empirical evidence supporting the use of BE to enhance health.

\subsection{Application of $B E$}

Health offers a number of different potential contexts for BE strategies to be implemented. These include individual lifestyle choices, government policy choices to promote public health and the decisions of physicians, patients and healthcare systems in medical care.

\subsubsection{Survey of the Application of BE to Individual Choice-Evidence from Childhood Obesity}

Extensive research has focused on the impact of BE interventions on children's food choices. Among the techniques that have been explored are messaging, choice and variety, social norms, incentives, convenience and defaults/substitutes to influence food choice in settings such as school cafeterias.

Madden and colleagues [20] argue that while standard educational approaches that use messaging to improve a child's awareness of nutritional principles are important, they do not produce a sustained improvement in dietary patterns. Messaging prompts such as simple signs encouraging consumption of water or white milk and direct verbal prompts from adult staff also have the power to significantly alter food decision-making at point-of-purchase [21-23]. Increasing the availability, variety and portion sizes of fruits and vegetables in relation to less healthy choices have also been shown to increase the selection of healthy items $[24,25]$. In a systematic review of behavioral economic interventions targeting child decision making in school cafeterias, Gordon et al. showed that interventions that relied on Kahneman's System 1 thinking (e.g., visual clues) were much more likely to be successful than those that relied on System 2 thinking (e.g., information comparison) [3].

Social norms can both positively and negatively influence dietary patterns. Peer modeling of both selection and preference for healthy choices can significantly influence consumption and preferences for these options by peers [26]. For example, when children are exposed to information about the vegetable intake of other children, greater consumption of vegetables is observed [27]. The normative influence of parental food choice can sway children from an early age. The healthfulness of childhood dietary choices is significantly correlated to the healthfulness of parental choice [28-30].

Monetary and small prize incentives have been used to improve food choices in children. Although there is significant debate surrounding the sustainability of monetary or small prize incentives in terms of their long-term cost and waning or negative effect once removed, both have been shown to be effective in increasing children's selection and consumption of healthy foods. Small monetary incentives significantly increase consumption of fruits and vegetables with increasing effect when larger monetary incentives were provided and when rewards were provided immediately [31,32]. The same effects are observed for prizes with regard to size and timing. Children of lower socioeconomic status were found to be more sensitive to these incentives compared to their peers of higher socioeconomic status. The positive effect of small prize incentives, however, decreases with time and is almost extinguished when the rewards are stopped $[33,34]$. The effect of decreasing opportunity cost of healthy choices has been explored as well. Increasing convenience of healthy food purchase by, for example, creating a separate salad bar or healthy food express line, can decrease the purchase of unhealthy foods and increase the purchase 
of healthy foods but may also increase total food waste [35,36]. Increasing convenience by pre-slicing fruits and vegetables has also been shown to increase consumption [37].

Default choices and intentional substitution of healthy for unhealthy options remove the child's ability to make an active choice in favor of a more paternalistic approach. Default interventions have included automatic placement of fruits and vegetables on lunch trays in school cafeterias and provision of default white milk (over chocolate milk). While interventions using default options have been shown to improve the selection of white milk, fruits and vegetables, there is an associated increase in food waste $[38,39]$. Substitution is a similar technique in which unhealthy options are replaced with healthier options either before the point of purchase or at the point of purchase. For example, one study examined the effect of replacing fruit for standard dessert options, which resulted in a cumulative increase in consumption of total servings of fruits and vegetables [40]. Allowing children to consume healthy options (e.g., sampling vegetables in line) before making additional choices is another form of substitution that has been found to increase the total consumption of fruits and vegetables [41]. Frank and colleagues provide helpful guidelines for employing $\mathrm{BE}$ to reduce childhood obesity in pediatric practice in response to aggressive marketing of unhealthy options to children [42].

\subsubsection{Survey of the Application of BE Government and Public Health}

$\mathrm{BE}$ is increasingly recognized as having a role in public health interventions [43]. The World Health Organization in their 2016 report on ending childhood obesity suggest a number of behavioral economic initiatives that could be instituted on a national/regional level [44]:

- Sugar-Sweetened Beverage Tax (SSBT);

- A set of recommendations to limit the types of advertising exposure in children and adolescents (specifically, they recommend that advertising of poor-quality foods not be geared to children and the use of cartoon characters be limited to healthy food items);

- Standardized worldwide nutritional labeling; and

- Interpretive front-of-pack food labeling

Taxes on sugar-sweetened beverages are now well-studied and typically found to decrease sugary drink purchases and increase government revenue [45]. Taxes on sugarsweetened beverages have the additive effect of the neoclassical economic element of increasing price leading to decreased purchasing and the behavioral element of loss aversion [46]. Berkeley, California was an early adopter of the SSBT in the USA [47]. After implementing the $\$ 0.01$ per ounce tax, sugar-sweetened beverage consumption dropped by $21 \%$, while there was a $4 \%$ increase in comparison cities. Water consumption increased by $63 \%$ while the tax generated $\$ 692,000$ for the city in the first six months of the intervention [48]. In addition to a tax on nonessential energy-dense foods, Mexico initiated a nationwide $10 \%$ SSBT, which resulted in a $12 \%$ decline in sugar-sweetened beverage purchases [49].

Direct advertising of unhealthy food items to children and adolescents leads to increased purchase of these items [50]. Several studies show that there is limited adherence by the food industry to voluntary codes restricting advertising of unhealthy foods to children and adolescents [51]. In response to concerns about direct marketing to youths, several countries have implemented advertising restrictions. The European Union has restrictions, including restrictions on cross-border marketing [52]. Although research on the effects of restrictions is limited, a study on restricted advertising to children in Singapore indicates that restrictions can improve outcomes [53]. Lwin and colleagues find that the restrictions did reduce unhealthy food advertising, the amount of unhealthy food in households decreased, and children's self-reported consumption of unhealthy food decreased modestly [53].

Several countries have introduced front-of-pack food labeling [54]. As expected, front-of-pack labeling that relies on System 1 thinking (identifies the product as healthy or unhealthy) tends to be more effective than labeling that relies on System 2 thinking (provides numerical nutritional information) [55]. An internationally accepted food labeling system as advocated by the World Health Organization has obvious appeal but still needs to be developed [44]. 
Chile's initiative to end obesity presents an interesting case study of a concerted nationwide effort employing BE. In 1960, Chile implemented a tax on all nonalcoholic beverages. In 1976, the tax was fixed at 13\%. In 2014, the tax was modified so that there was a $5 \%$ increase in the tax on sugar-sweetened beverages and a decrease of $3 \%$ on soft drinks with low sugar levels [56]. Additionally, in 2016, as noted by Pérez-Escamilla, Lutter et al., Chile began a three-year, stepwise implementation that included [57]:

- Point-of-purchase food labeling with easy-to-understand information on sugar, saturated fat and calorie content;

- Restriction on advertising and sales of unhealthy foods to children; and

- Interpretive front-of-pack food labeling.

While the health effects of this national intervention are still being evaluated, the food industry is responding with more low-sugar drinks and healthier food options [58].

Although our main focus has been on obesity, BE has also been applied to understanding substance abuse and addiction (hyperbolic discounting) and to offer strategies for recovery. Briefly, the main guidance from BE is to make the desired choice easier by increasing costs associated with the abusive behavior and the rewards of substance-free choices and decreasing potential (legal) penalties for seeking treatment. In a longitudinal study, DeCicca and colleagues find that state-level cigarette taxes increase costs of smoking and are associated with cessation of smoking but have limited impact on initiation [59]. Work by Nonnemaker and Farrelly found an initiation effect of increasing the cost of cigarettes, especially for Black youths [60]. Similar guidance to curb alcohol abuse on college campuses focuses on increasing the monetary and behavioral cost of such behaviors while increasing the rewards of substance-free activities [61]. Harm reduction in seeking treatment (referral to treatment rather than incarceration) is another strategy to incentivize the desired behavior as it relates to drug policy [62].

3.2.3. Survey of the Application of BE in Medicine: Heuristics and Bias, the Negative Side of Behavioral Economics

Medical decision-making, as well as other decision-making as noted in the BE discussion above, is often influenced by cognitive bias and the use of heuristics. These influences can cause cognitive error by health care users, medical practitioners, and health policy designers [63]. Understanding these pitfalls could potentially lead to correcting negative influences and result in better outcomes [64]. Possible cognitive mishaps include the following $[63,64]$.

Representativeness Heuristic: This occurs when we draw conclusions based on the memory of a prototype, stereotype or average. In this situation, baseline rates of occurrence are often ignored and we defer to something that is less likely but fits an expected pattern [65].

Availability Bias: This is a bias or heuristic in which we make a decision based on recent experiences or other memorable exposures. For example, a doctor may be more prone to make a diagnosis of pulmonary fibrosis if he or she recently saw a case of that disorder [65].

Optimism Bias: People tend to be very optimistic and believe they are less likely to experience a negative event than others. This bias may interfere with individuals seeking medical care or making appropriate health decisions because they minimize their risk [66].

Confirmation Bias: This is the tendency to look for facts or information that confirms a hypothesis or belief and can lead to cognitive errors for medical practitioners, health care workers, and public health planners [67-69].

Illusory Correlation: This is viewing two entities as related when they actually may be occurring together merely out of chance and are not related at all [70]. This commonly occurs in patient and medical practitioner decisions when symptoms and disease may be attributed to an irrelevant diagnostic finding.

Ikea Effect: This is a cognitive bias in which we tend to favor things that we create or partially create [71]. This may be cherishing a desk that we assemble, hence the term, 
"Ikea Effect", or an idea such as a diagnosis. Thus, we may give undue weight to things we create in decision making.

While these biases often lead to cognitive error, they can at times lead to decisions or actions that improve health. One example is when school-aged children are asked to create a "peacock" with vegetables, they were more likely to eat the vegetables [72]. Still, it is important for us to be aware of these biases in medical and health policy decision making and look for information that challenges our assumptions [63].

\subsection{Using BE to Improve Health and Well-Being by Improving Medical Practice}

$\mathrm{BE}$ in the form of neoclassical incentives and nudging has great potential to improve medical decision making and health care [43].

Incentives have been used to modify behavior in a variety of settings including healthcare [73]. In a randomized controlled study of isoniazid prophylaxis among the homeless in San Francisco, Tulsky and colleagues showed almost 100\% improvement in adherence when a five-dollar incentive was given to participants who took their medication as compared to no incentive [74]. In an effort to lower obesity rates in Dubai, the emirate offered individuals one gram of gold for every kilogram of weight loss. Over 5000 people participated in the program [75]. Incentives have been used to improve physician performance with mixed results. While practitioner bonuses improved childhood immunization rates in the United Kingdom from 70 to $90 \%$, a systematic review of monetary incentives showed modest to no improvement in immunization and cancer screening rates in the United States [76] (Town, Kane et al. 2005). It is not surprising, however, that behavior change prompted by incentives does not appear to be longstanding. When the incentives are stopped, the effect often dissipates [74].

Defaults, with an ability to opt out, are an effective type of nudge. Accepting the desirable path is passive. With this type of intervention, the desirable result is set as the default. To choose another option, an individual must "opt out" and actively choose another option. This has been used successfully in several countries to improve organ donation rates [77]. Patel and colleagues set generic drugs as the default choice in their electronic health record ordering system. They were able to increase generic drug prescription rate by $75 \%$ [78]. One potential downside, however, to opt-out default systems is that they may lead to over-ordering of diagnostic tests when users have limited capacity to override the default options [79].

The "peak end rule" is a heuristic that potentially can lead to a better patient experience. Redelmeier demonstrated that individuals may tolerate a more unpleasant experience if at the end there is less discomfort than another experience with a lower average level of discomfort [80]. Kahneman's group tested this effect in a randomized trial in which half of patients undergoing colonoscopy had a short segment at the end of the procedure in which patients experienced less discomfort than under the traditional procedure. Those that had the added interval to their colonoscopy were 10\% more likely to return for a follow-up colonoscopy [80].

While anchoring, representativeness and availability heuristics could potentially be used to influence decision making in a positive manner, most studies have focused on the negative aspects such as decision-making errors by medical practitioners and healthcare users and efforts to recognize and eliminate them [81-83]. Educational efforts to recognize bias may be helpful. In addition, efforts to slow down the medical diagnostic process with formal protocols that list diagnostic alternatives may be helpful. Unfortunately, interventions to reduce these three biases have been mixed [84] (O'Sullivan and Schofield 2018). Jenkins successfully used an education module to help physicians recognize these biases, which led to fewer diagnostic errors in treating pediatric bipolar disorder [80]. Mamede was able to reduce diagnostic error by internal medicine residents by $15 \%$ in first-year residents and $24 \%$ in second-year residents with a formal diagnostic protocol to address decision making bias [85]. Experimentally, Lee and colleagues used an educational game to eliminate anchoring, availability and representativeness biases, but the effect was 
extinguished after four weeks [86]. Navathe and colleagues (2020) find a positive impact on the quality of care provided by the use of peer comparison feedback, a BE intervention, in a cluster-randomized controlled trial with Blue Cross Blue Shield of Hawaii. However, further research is needed to address and to mitigate these biases [87].

\subsection{Sustainability}

Our surveys of the BE intervention in healthcare point to several observations. The first is that, as Gordon and colleagues and others have found, System 1 is a more effective tool to support healthy decision making by children in cafeterias and in broader society-level decision making when information is provided for food items [3,54,55]. Similarly, medical decision-making on the part of both healthcare professionals and patients can be improved through the use of strategies such as default and opt-out systems. While previous research has focused on the risk of cognitive error when heuristics and other forms of cognitive bias are used in medical decision-making, there is emerging evidence demonstrating the potential benefit of targeting cognitive biases to optimize choices. Furthermore, healthy decisions can be reinforced by using taxes to alter price signals in pursuit of public health goals. Using bonuses and prices to incentivize choices tends to be less effective and less sustainable due to both the size of needed incentives and the ongoing costs of maintaining the incentives. If the incentives are not maintained, the behaviors tend to revert to the initial less desirable ones.

In addition to BE strategies applied directly to the health decisions outlined above, the strategies associated with BE can be employed in support of the objectives identified in the UN statement on sustainable development goals, "Achieving the SDGs requires the partnership of governments, private sector, civil society and citizens alike to make sure we leave a better planet for future generations" (https://www.undp.org/content/undp/en/ home/sustainable-development-goals.html (accaessed on 1 June 2021)).

BE strategies can support the health-related sustainable development goals in several ways. First, by guiding individual choices to avoid unhealthy behaviors, a number of the specific 13 targets associated with UNSDG 3 will be directly impacted, including (1) ending the epidemics of AIDS, tuberculosis, malaria and neglected tropical diseases; (2) combating hepatitis, waterborne diseases and other communicable diseases; (3) halving the number of global deaths and injuries from road traffic accidents; (4) strengthening the prevention and treatment of substance abuse, including narcotic drug abuse and harmful use of alcohol; and (5) strengthening the implementation of the World Health Organization Framework Convention on Tobacco Control in all countries.

A reduction in the burden of chronic conditions through improved personal choice through $\mathrm{BE}$ combined with the reduction in medical errors by application of $\mathrm{BE}$ to the practice of medicine will free up resources in the health care system to support additional sustainable development health goals. Among the goals to be achieved by 2030 are (1) the reduction the global maternal mortality ratio to less than 70 per 100,000 live births; (2) the end preventable deaths of newborns and children under five years of age; (3) ensuring universal access to sexual and reproductive health-care services, including for family planning, information and education, and the integration of reproductive health into national strategies and programs; (4) achieving universal health coverage, including financial risk protection, access to quality essential health-care services and access to safe, effective, quality and affordable essential medicines and vaccines for all; (5) substantially reducing the number of deaths and illnesses from hazardous chemicals and air, water and soil pollution and contamination; (6) supporting the research and development of vaccines and medicines for the communicable and noncommunicable diseases that primarily affect developing countries, provide access to affordable essential medicines and vaccines; (7) substantially increasing health financing and the recruitment, development, training and retention of the health workforce in developing countries, especially in least-developed countries and small island developing states; and (8) strengthening the capacity of all countries, in particular developing countries, for early warning, 
risk reduction and management of national and global health risks (targets from WHO https://www.who.int/health-topics/sustainable-development-goals\#tab=tab_2 (accessed on 1 June 2021)).

Since BE interventions are largely based on heuristics and nudging people to make better decisions, they are by their very nature cost-effective and sustainable [46]. Thus, it is not surprising that countries with somewhat limited resources such as many countries in Latin America are instituting policies based on behavioral economic principles such as soda taxes and front-of-pack food labeling [57]. Even interventions with monetary incentives that have significant upfront costs ultimately can be cost-saving through better health outcomes [88]. Taxing unhealthy food items, a neoclassical-based intervention, not only leads to healthier lifestyles but very quickly increases revenues which can be used for additional health policy, or other pressing initiatives [89].

Not only are BE initiatives likely to be self-sustaining, they can also be instrumental in the sustainability of the planet. In a Lancet Commission Report, Swinburn and colleagues describe the global syndemic of obesity, undernutrition and climate change [90]. The report recognizes that all three global problems are powered by the same drivers: cheap fossil fuels and ultra-processed food [90]. Obesity, undernutrition and climate change also reinforce each other and disproportionally affect poorer nations and the poor within richer nations. It is predicted that over 70 percent of deaths due to non-communicable diseases will occur in lower-income countries [91] (GBD Obesity Collaborators 2017) despite poorer nations contributing less greenhouse gases than more affluent countries. Given that behavioral economic initiatives have been demonstrated to be low in cost and effectively lead to a healthier lifestyle, they may play a key role in battling climate change. Harnessing the power of $\mathrm{BE}$ is one strategy to promote and achieve the sustainable goal of health and well-being for all.

Funding: There was no external funding for this project.

Institutional Review Board Statement: Not applicable as this project did not involve the study human subjects but rather a review of the existing literature.

Informed Consent Statement: Not applicable.

Data Availability Statement: Not applicable.

Conflicts of Interest: The authors declare no conflict of interest.

\section{References}

1. Aldieri, L.; Bruno, B.; Vinci, C.P. A multi-dimensional approach to happiness and innovation. Appl. Econ. 2021, 53, 1300-1310. [CrossRef]

2. Usai, A.; Orlando, B.; Mazzoleni, A. Happiness as a Driver of Entrepreneurial Initiative and Innovation Capital. J. Intellect. Cap. 2020, 21, 1229-1255. [CrossRef]

3. Gordon, K.; Dynan, L.; Siegel, R. Healthier choices in school cafeterias: A systematic review of cafeteria interventions. J. Pediatrics 2018, 203, 273-279. [CrossRef]

4. Dhami, S. The Foundations of Behavioral Economic Analysis; Oxford University Press: Oxford, UK, 2016.

5. Bucher, T.; Collins, C.; Rollo, M.E.; McCaffrey, T.A.; De Vlieger, N.; Van der Bend, D.; Truby, H.; Perez-Cueto, F.J. Nudging consumers towards healthier choices: A systematic review of positional influences on food choice. Br. J. Nutr. 2016, 115, 2252-2263. [CrossRef]

6. Stanton, R.A. Food retailers and obesity. Curr. Obes. Rep. 2015, 4, 54-59. [CrossRef]

7. Mischel, W.; Ebbesen, E.B.; Raskoff Zeiss, A. Cognitive and attentional mechanisms in delay of gratification. J. Personal. Soc. Psychol. 1972, 21, 204. [CrossRef]

8. Morrison, W.; Taubinsky, D. Rules of Thumb and Attention Elasticities: Evidence from Under-and Overreaction to Taxes; National Bureau of Economic Research: Cambridge, MA, USA, 2019.

9. Duckworth, A.L.; Taxer, J.L.; Eskreis-Winkler, L.; Galla, B.M.; Gross, J.J. Self-control and academic achievement. Annu. Rev. Psychol. 2019, 70, 373-399. [CrossRef]

10. Thaler, R.H.; Sunstein, C.R. Nudge: Improving Decisions About Health, Wealth, and Happiness; Yale University Press: New Haven, CT, USA, 2008.

11. Kahneman, D. Thinking, Fast and Slow; Macmillan Ltd.: Basingstoke, UK, 2011.

12. Kahneman, D.; Tversky, A. Subjective probability: A judgment of representativeness. Cogn. Psychol. 1972, 3, 430-454. [CrossRef] 
13. Jenssen, B.P.; Buttenheim, A.M.; Fiks, A.G. Using Behavioral Economics to Encourage Parent Behavior Change: Opportunities to Improve Clinical Effectiveness. Acad. Pediatr. 2019, 19, 4-10. [CrossRef]

14. Thaler, R. Some empirical evidence on dynamic inconsistency. Econ. Lett. 1981, 8, 201-207. [CrossRef]

15. Camerer, C.F. Prospect Theory in the Wild: Evidence from the Field; Working Papers: No 1037; California Institute of Technology, Division of the Humanities and Social Sciences: Pasadena, CA, USA, 1998; pp. 148-161.

16. Plott, C.R.; Zeiler, K. Exchange asymmetries incorrectly interpreted as evidence of endowment effect theory and prospect theory? Am. Econ. Rev. 2007, 97, 1449-1466. [CrossRef]

17. Bhattacharya, J.; Hyde, T.; Tu, P. Health Economics; Macmillan International Higher Education: New York, NY, USA, 2013.

18. Pesendorfer, W. Behavioral economics comes of age: A review essay on advances in behavioral economics. J. Econ. Lit. 2006, 44, 712-721. [CrossRef]

19. Kelman, M. Behavioral economics as part of a rhetorical duet: A response to Jolls, Sunstein, and Thaler. Stan. L. Rev. 1997, 50, 1577. [CrossRef]

20. Madden, G.J.; Price, J.; Sosa, F.A. Behavioral economic approaches to influencing children's dietary decision making at school. Policy Insights Behav. Brain Sci. 2017, 4, 41-48. [CrossRef]

21. Schwartz, M.B. The influence of a verbal prompt on school lunch fruit consumption: A pilot study. Int. J. Behav. Nutr. Phys. Act. 2007, 4, 6. [CrossRef]

22. Kenney, E.L.; Gortmaker, S.L.; Carter, J.E.; Howe, M.C.W.; Reiner, J.F.; Cradock, A.L. Grab a cup, fill it up! An intervention to promote the convenience of drinking water and increase student water consumption during school lunch. Am. J. Public Health 2015, 105, 1777-1783. [CrossRef]

23. Lai, C.-Y.; List, J.A.; Samek, A. How Do Informational Prompts Affect Choices in the School Lunchroom? CESR-Schaeffer Working Paper (2017-001). 2017. Available online: https:/ / papers.ssrn.com/sol3/papers.cfm?abstract_id=2909407 (accessed on 1 June 2021).

24. Hakim, S.M.; Meissen, G. Increasing consumption of fruits and vegetables in the school cafeteria: The influence of active choice. J. Health Care Poor Underserved 2013, 24, 145-157. [CrossRef]

25. Miller, N.; Reicks, M.; Redden, J.P.; Mann, T.; Mykerezi, E.; Vickers, Z. Increasing portion sizes of fruits and vegetables in an elementary school lunch program can increase fruit and vegetable consumption. Appetite 2015, 91, 426-430. [CrossRef]

26. Houldcroft, L.; Haycraft, E.; Farrow, C. Peer and friend influences on children's eating. Soc. Dev. 2014, 23, 19-40. [CrossRef]

27. Sharps, M.; Robinson, E. Perceived eating norms and vegetable consumption in children. Int. J. Behav. Nutr. Phys. Activity 2015, 12, 135. [CrossRef]

28. Epstein, L.H.; Dearing, K.K.; Handley, E.A.; Roemmich, J.N.; Paluch, R.A. Relationship of mother and child food purchases as a function of price: A pilot study. Appetite 2006, 47, 115-118. [CrossRef]

29. Epstein, L.H.; Dearing, K.K.; Paluch, R.A.; Roemmich, J.N.; Cho, D. Price and maternal obesity influence purchasing of low-and high-energy-dense foods. Am. J. Clin. Nutr. 2007, 86, 914-922. [CrossRef]

30. Sutherland, L.A.; Beavers, D.P.; Kupper, L.L.; Bernhardt, A.M.; Heatherton, T.; Dalton, M.A. Like parent, like child: Child food and beverage choices during role playing. Arch. Pediatrics Adolesc. Med. 2008, 162, 1063-1069. [CrossRef]

31. Just, D.; Price, J. Default options, incentives and food choices: Evidence from elementary-school children. Public Health Nutr. 2013, 16, 2281-2288. [CrossRef] [PubMed]

32. List, J.A.; Samek, A.S. The behavioralist as nutritionist: Leveraging behavioral economics to improve child food choice and consumption. J. Health Econ. 2015, 39, 135-146. [CrossRef]

33. Siegel, R.; Lockhart, M.K.; Barnes, A.S.; Hiller, E.; Kipp, R.; Robison, D.L.; Ellsworth, S.C.; Hudgens, M.E. Small prizes increased healthful school lunch selection in a Midwestern school district. Appl. Physiol. Nutr. Metab. 2015, 41, 370-374. [CrossRef] [PubMed]

34. Emerson, M.; Hudgens, M.; Barnes, A.; Hiller, E.; Robison, D.; Kipp, R.; Bradshaw, U.; Siegel, R. Small prizes increased plain milk and vegetable selection by elementary school children without adversely affecting total milk purchase. Beverages $\mathbf{2 0 1 7}, 3,14$. [CrossRef]

35. Hanks, A.S.; Just, D.R.; Smith, L.E.; Wansink, B. Healthy convenience: Nudging students toward healthier choices in the lunchroom. J. Public Health 2012, 34, 370-376. [CrossRef]

36. Adams, M.A.; Bruening, M.; Ohri-Vachaspati, P.; Hurley, J.C. Location of school lunch salad bars and fruit and vegetable consumption in middle schools: A cross-sectional plate waste study. J. Acad. Nutr. Diet. 2016, 116, 407-416. [CrossRef]

37. Wansink, B.; Just, D.R.; Hanks, A.S.; Smith, L.E. Pre-sliced fruit in school cafeterias: Children's selection and intake. Am. J. Prev. Med. 2013, 44, 477-480. [CrossRef]

38. Just, D.R.; Price, J. Using incentives to encourage healthy eating in children. J. Human Resour. 2013, 48, 855-872.

39. Hanks, A.S.; Just, D.R.; Wansink, B. Chocolate milk consequences: A pilot study evaluating the consequences of banning chocolate milk in school cafeterias. PLoS ONE 2014, 9, e91022. [CrossRef]

40. Zellner, D.A.; Cobuzzi, J.L. Just dessert: Serving fruit as a separate dessert course increases vegetable consumption in a school lunch. Food Qual. Prefer. 2016, 48, 195-198. [CrossRef]

41. Elsbernd, S.; Reicks, M.M.; Mann, T.L.; Redden, J.P.; Mykerezi, E.; Vickers, Z.M. Serving vegetables first: A strategy to increase vegetable consumption in elementary school cafeterias. Appetite 2016, 96, 111-115. [CrossRef] [PubMed] 
42. Frank, H.R.; Ubel, P.A.; Wong, C.A. Behavioral economic insights for pediatric obesity: Suggestions for translating the guidelines for our patients. JAMA Pediatrics 2020, 174, 319-320. [CrossRef]

43. Hursh, S.R.; Roma, P.G. Behavioral economics and empirical public policy. J. Exp. Anal. Behav. 2013, 99, 98-124. [CrossRef]

44. World Health Organization. Consideration of the Evidence on Childhood Obesity for the Commission on Ending Childhood Obesity: Report of the Ad Hoc Working Group on Science and Evidence for Ending Childhood Obesity; World Health Organization: Geneva, Switzerland, 2016.

45. Brownell, K.D.; Farley, T.; Willett, W.C.; Popkin, B.M.; Chaloupka, F.J.; Thompson, J.W.; Ludwig, D.S. The public health and economic benefits of taxing sugar-sweetened beverages. N. Engl. J. Med. 2009, 361, 1599. [CrossRef]

46. Galizzi, M.M. Label, nudge or tax? A review of health policies for risky behaviours. J. Public Health Res. 2012, 1, 14. [CrossRef] [PubMed]

47. Falbe, J.; Thompson, H.R.; Becker, C.M.; Rojas, N.; McCulloch, C.E.; Madsen, K.A. Impact of the Berkeley excise tax on sugar-sweetened beverage consumption. Am. J. Public Health 2016, 106, 1865-1871. [CrossRef]

48. Ng, S.W.; Silver, L.; Ryan-Ibarra, S.; Induni, M.; Hamma, C.; Poti, J.; Popkin, B. Berkeley evaluation of soda tax (BEST) study preliminary findings. In Proceeding of the Presentation at the Annual Meeting of the American Public Health Association, Chicago, IL, USA, 3 November 2015.

49. Colchero, M.A.; Popkin, B.M.; Rivera, J.A.; Ng, S.W. Beverage purchases from stores in Mexico under the excise tax on sugar sweetened beverages: Observational study. BMJ 2016, 352, h6704. [CrossRef]

50. Caraher, M.; Landon, J. The impact of advertising on food choice: The social context of advertising. Front. Nutr. Sci. $2006,3,22$.

51. Galbraith-Emami, S.; Lobstein, T. The impact of initiatives to limit the advertising of food and beverage products to children: A systematic review. Obes. Rev. 2013, 14, 960-974. [CrossRef] [PubMed]

52. World Health Organization. A Framework for Implementing the Set of Recommendations on the Marketing of Foods and Non-Alcoholic Beverages to Children; World Health Organization: Geneva, Switzerland, 2012.

53. Lwin, M.O.; Yee, A.Z.; Lau, J.; Ng, J.S.; Lam, J.Y.; Cayabyab, Y.M.; Vijaya, K. A macro-level assessment of introducing children food advertising restrictions on children's unhealthy food cognitions and behaviors. Int. J. Advert. 2020, 39, 990-1011. [CrossRef]

54. Kelly, B.; Jewell, J. What is the Evidence on the Policy Specifications, Development Processes and Effectiveness of Existing Front-of-Pack food Labelling Policies in the WHO European Region? World Health Organization: Geneva, Switzerland, 2018.

55. Talati, Z.; Pettigrew, S.; Kelly, B.; Ball, K.; Dixon, H.; Shilton, T. Consumers' responses to front-of-pack labels that vary by interpretive content. Appetite 2016, 101, 205-213. [CrossRef]

56. Caro, J.C.; Ng, S.W.; Taillie, L.S.; Popkin, B.M. Designing a tax to discourage unhealthy food and beverage purchases: The case of Chile. Food Policy 2017, 71, 86-100. [CrossRef]

57. Pérez-Escamilla, R.; Lutter, C.; Rabadan-Diehl, C.; Rubinstein, A.; Calvillo, A.; Corvalán, C.; Batis, C.; Jacoby, E.; Vorkoper, S.; Kline, L. Prevention of childhood obesity and food policies in Latin America: From research to practice. Obes. Rev. 2017, 18, 28-38. [CrossRef]

58. Jacobs, A. In Sweeping War on Obesity, Chile Slays Tony the Tiger. New York times. 2018. Available online: https://www. nytimes.com/2018/02/07/health/obesity-chile-sugar-regulations.html?searchResultPosition=1 (accessed on 10 August 2019).

59. DeCicca, P.; McLeod, L. Cigarette taxes and older adult smoking: Evidence from recent large tax increases. J. Health Econ. 2008, 27, 918-929. [CrossRef] [PubMed]

60. Nonnemaker, J.M.; Farrelly, M.C. Smoking initiation among youth: The role of cigarette excise taxes and prices by race/ethnicity and gender. J. Health Econ. 2011, 30, 560-567. [CrossRef]

61. Murphy, J.G.; Correia, C.J.; Barnett, N.P. Behavioral economic approaches to reduce college student drinking. Addict. Behave. 2007, 32, 2573-2585. [CrossRef]

62. Marlatt, G.A.; Kilmer, J.R. Consumer choice: Implications of behavioral economics for drug use and treatment. Behav. Ther. 1998, 29, 567-576. [CrossRef]

63. Klein, J.G. Five pitfalls in decisions about diagnosis and prescribing. BMJ 2005, 330, 781-783. [CrossRef]

64. Smith, B.W.; Slack, M.B. The effect of cognitive debiasing training among family medicine residents. Diagnosis 2015, 2, 117-121. [CrossRef]

65. Tversky, A.; Kahneman, D. Judgment under uncertainty: Heuristics and biases. Science 1974, 185, 1124-1131. [CrossRef] [PubMed]

66. Sharot, T. The optimism bias. Curr. Biol. 2011, 21, R941-R945. [CrossRef] [PubMed]

67. Chapman, G.B.; Elstein, A.S. Cognitive processes and biases in medical decision making. Decision Making in Health Care: Theory, Psychology, and Applications; Cambridge University Press: Cambridge, UK, 2000; pp. 183-210.

68. Keselman, A.; Browne, A.C.; Kaufman, D.R. Consumer health information seeking as hypothesis testing. J. Am. Med. Inform. Assoc. 2008, 15, 484-495. [CrossRef] [PubMed]

69. Cheung, R.; Ardolino, A. Behavioural science in public health policy. Br. J. Healthc. Manag. 2011, 17, 140-144. [CrossRef]

70. Gorini, A.; Pravettoni, G. An overview on cognitive aspects implicated in medical decisions. Eur. J. Intern. Med. 2011, 22, 547-553. [CrossRef] [PubMed]

71. Norton, M.I.; Mochon, D.; Ariely, D. The IKEA effect: When labor leads to love. J. Consum. Psychol. 2012, 22, 453-460. [CrossRef]

72. Raghoebar, S.; van Kleef, E.; de Vet, E. Self-crafting vegetable snacks: Testing the IKEA-effect in children. Br. Food J. 2017, 119, 1301-1312. [CrossRef] 
73. Gneezy, U.; Meier, S.; Rey-Biel, P. When and why incentives (don't) work to modify behavior. J. Econ. Perspect. 2011, 25, 191-210. [CrossRef]

74. Tulsky, J.P.; Pilote, L.; Hahn, J.A.; Zolopa, A.J.; Burke, M.; Chesney, M.; Moss, A.R. Adherence to isoniazid prophylaxis in the homeless: A randomized controlled trial. Arch. Intern. Med. 2000, 160, 697-702. [CrossRef]

75. Venkatapuram, S. There Are Many Purposes for Conditional Incentives to Accessing Healthcare Comment on Denial of Treatment to Obese Patients—-the Wrong Policy on Personal Responsibility for Health. Int. J. Health Policy Manag. 2013, 1, 235. [CrossRef]

76. Town, R.; Kane, R.; Johnson, P.; Butler, M. Economic incentives and physicians' delivery of preventive care: A systematic review. Am. J. Prev. Med. 2005, 28, 234-240. [CrossRef]

77. Saab, S.; Saggi, S.S.; Akbar, M.; Choi, G. Presumed consent: A potential tool for countries experiencing an organ donation crisis. Digest. Dis. Sci. 2019, 64, 1346-1355. [CrossRef]

78. Patel, M.S.; Day, S.C.; Halpern, S.D.; Hanson, C.W.; Martinez, J.R.; Honeywell, S.; Volpp, K.G. Generic medication prescription rates after health system-wide redesign of default options within the electronic health record. JAMA Intern. Med. 2016, 176, 847-848. [CrossRef]

79. Ansher, C.; Ariely, D.; Nagler, A.; Rudd, M.; Schwartz, J.; Shah, A. Better medicine by default. Med Decis. Mak. 2014, 34, 147-158. [CrossRef] [PubMed]

80. Redelmeier, D.A.; Katz, J.; Kahneman, D. Memories of colonoscopy: A randomized trial. Pain 2003, 104, 187-194. [CrossRef]

81. Brezis, M.; Halpern-Reichert, D.; Schwaber, M.J. Mass Media-Induced Availability Bias in the Clinical Suspicion of West Nile Fever. Ann. Intern. Med. 2004, 140, 234-235. [CrossRef] [PubMed]

82. Elia, F.; Apra, F.; Verhovez, A.; Crupi, V. First, know thyself: Cognition and error in medicine. Acta. Diabetol. 2016, 53, 169-175. [CrossRef]

83. Cho, I.; Bates, D.W. Behavioral economics interventions in clinical decision support systems. Yearb. Med Inform. 2018, $27,114$. [CrossRef]

84. O'Sullivan, E.; Schofield, S. Cognitive bias in clinical medicine. J. R. Coll Physicians Edinb. 2018, 48, 225-232. [CrossRef]

85. Mamede, S.; van Gog, T.; van den Berge, K.; Rikers, R.M.; van Saase, J.L.; van Guldener, C.; Schmidt, H.G. Effect of availability bias and reflective reasoning on diagnostic accuracy among internal medicine residents. JAMA 2010, 304, 1198-1203. [CrossRef]

86. Lee, Y.-H.; Dunbar, N.E.; Miller, C.H.; Lane, B.L.; Jensen, M.L.; Bessarabova, E.; Burgoon, J.K.; Adame, B.J.; Valacich, J.J.; Adame, E.A. Training anchoring and representativeness bias mitigation through a digital game. Simul. Gaming 2016, 47, 751-779. [CrossRef]

87. Navathe, A.S.; Volpp, K.G.; Bond, A.M.; Linn, K.A.; Caldarella, K.L.; Troxel, A.B.; Zhu, J.; Yang, L.; Matloubieh, S.E.; Drye, E.E.; et al. Assessing the Effectiveness Of Peer Comparisons As A Way To Improve Health Care Quality. Health Aff. 2020, 39, 852-861. [CrossRef] [PubMed]

88. Lesser, L.I.; Puhl, R.M. Alternatives to monetary incentives for employee weight loss. Am. J. Prev. Med. 2014, 46, 429-431. [CrossRef] [PubMed]

89. Roache, S.A.; Gostin, L.O. The untapped power of soda taxes: Incentivizing consumers, generating revenue, and altering corporate behavior. Int. J. Health Policy Manag. 2017, 6, 489. [CrossRef]

90. Swinburn, B. Planet Obesity: How we're Eating Ourselves and the Planet to Death. 2011. Available online: ReadHow YouWant.com (accessed on 1 June 2021).

91. GBD Obesity Collaborators. Health effects of overweight and obesity in 195 countries over 25 years. N. Engl. J. Med. 2017, 377, 13-27. [CrossRef] 